\title{
Filologia digitale (a partire dal lavoro per l'edizione informatica dello Zibaldone Laurenziano di Boccaccio)
}

\author{
Raul Mordenti, Università di Roma "Tor Vergata"
}

\begin{abstract}
The transformation of the text from the pre-information technology and Gutenberg modes to the model marked by information or digital technology is such that it substantially changes not only the concept of the text but also the nature of philology itself. This paper presents and discusses the problems encountered in producing a digital edition of the Zibaldone Laurenziano, Giovanni Boccaccio's handwritten manuscript conserved in the Laurenziana Library in Florence (Pluteo XXIX, 8). The Medieval text in general, and even more with the case of a zibaldone-type text, has intrinsic characteristics that clash with the immobility and definitive nature typical of the print text. The digital edition has been defined by Lebrave as the "critical edition of the 21st century." It can be based on hypertextuality and hypermediality, using the internet as a resource, and is perfectly able to render textual movement, restoring the text to its specific mobility.
\end{abstract}

Il passaggio del testo dalla modalità pre-informatica e gutemberghiana, a quella segnata dall'informatica o digitale è tale da cambiare sostanzialmente non solo il concetto di testo ma anche la natura stessa della filologia. Il saggio presenta e discute i problemi incontrati nel lavoro volto a stabilire un'edizione critica informatizzata dello Zibaldone Laurenziano, manoscritto autografo di Giovanni Boccaccio, conservato alla Biblioteca Laurenziana di Firenze (Pluteo XXIX, 8). Il testo medievale (e ancor più un testo del tipozibaldone) presenta delle caratteristiche intrinseche che entrano in contraddizione con l'immobilità e la natura definitiva tipiche del testo a stampa. L'edizione informatizzata, quella che si può basare sull'ipertestualità, sull'ipermedialità e sul ricorso alla rete, quella che Jean-Louis Lebrave ha definito come "l'édition critique au XXI.e siècle" (Lebrave 127), è perfettamente in grado di rendere il movimento testuale, restituendo al testo la sua rigorosa mobilità.

\section{A proposito del nesso fra le tecnologie del testo, le diverse idee di testo e $\mathbf{i}$ testi in quanto tali}

La tesi che vorrei argomentare è la seguente: il passaggio del testo dalla modalità preinformatica, che ormai possiamo definire gutemberghiana, a quella segnata dall'informatica o digitale è tale da cambiare sostanzialmente il concetto di testo, e dunque - direttamente la disciplina che verte sul testo in quanto tale, la filologia. 
Il presupposto di tale argomentazione è che esista un nesso profondo fra le modalità in cui il testo vive (e più precisamente le tecnologie che veicolano il testo) e il testo stesso: le tecnologie del testo (e anche l'oralità o la chirografia sono naturalmente da intendersi in questo ambito di ragionamento come tecnologie) non sono affatto vernici che ricoprono ex post il testo, non sono qualcosa che viene dopo il testo, ma - al contrario - sono un tutt'uno con il suo costituirsi e trasmettersi, cioè con il suo essere testo.

Noi abbiamo attraversato in passato almeno tre tornanti epocali nella storia della testualità umana, a cui se ne aggiunge ora un quarto: (a) l'oralità pura o assoluta (quella che affidava alla sola memoria la stabilità del testo, cioè la sua conservazione e trasmissione); (b) il passaggio dall'oralità alla scrittura chirografica e l'insediarsi della scrittura/lettura come medium di conservazione/trasmissione di messaggi (di cui è testimone un densissimo e celebre passo del Fedro di Platone); (c) il passaggio dalla chirografia alla stampa, come modalità dominante (e modellizzante) della testualità; e ora si svolge sotto i nostri occhi un quarto passaggio, (d) quello dalla «Galassia di Gutenberg» all'informatica.

Naturalmente, come sempre accade nella storia dei media, la novità non sopprime affatto ciò che l'ha preceduta, e dunque modalità diverse possono coesistere e coesistono anche molto a lungo: esattamente come i cavalli possono convivere con il dominio dell'automobile, anche la testualità orale sopravvisse (e sopravvive tutt'oggi), e i manoscritti convissero a lungo - e anzi battagliarono a lungo! - con le stampe, e così via. Ma ciò che conta è la dominanza, cioè il fatto che di volta in volta ci sia una modalità, e una sola, che domina su tutte le altre, che fornisce un modello anche teorico di testualità. Ed è nei tornanti che si vede più lontano e più chiaro. In questo senso, (almeno in questo senso) questa è una generazione fortunata, che non solo può vivere direttamente una rivoluzione del presente e del futuro ma che può anche vedere più chiaramente delle altre nel passato dell'umanità.

Come abbiamo affermato poc'anzi, esiste un nesso profondo fra le modalità tecnologiche in cui il testo vive, le diverse idee di testo che sorgono su tali basi e i testi in quanto tali. Ad esempio, ora ci appare chiaro - comincia ad apparirci chiaro, se solo vogliamo vederlo quanto il testo orale (propriamente orale) contenga in sé le marcature della necessità della memorizzazione (pensiamo ad esempio al carattere formulaico del testo omerico e, più avanti, alla stessa rima, la quale è da intendersi come traccia e sopravvivenza della memorabilità orale); e, ancora, cominciamo ora a vedere che la caratteristica e inesauribile mouvance riconosciuta nel testo manoscritto medievale (ma non riducibile ad esso soltanto) fosse in realtà un aspetto legato alla modalità della continua copiatura/riscrittura/glossatura caratteristica della chirografia; e per quanto riguarda la stampa ci appare ora più chiara la sua effettiva natura e consistenza storica di complessiva modalità di organizzazione e modellizzazione del sapere sociale, dunque il suo essere una epistemologia prima ancora che una tecnologia.

Tale caratteristica forte, cioè essere una complessiva epistemologia capace di riorganizzare complessivamente il sapere umano prima ancora che una potente tecnologia, deve essere riconosciuta da parte nostra anche all'informatica; questa dunque credo che 
debba interessarci anzitutto nei suoi aspetti teorici, e nelle sue conseguenze teoriche sull'idea che abbiamo del testo, cioè a prescindere dalla macchina informatica e dalla sue spettacolari capacità. Queste ultime anzi possono in un certo senso portarci fuori strada come accade nei convegni di informatica umanistica quando assistiamo a spettacolari sons et lumières (che appartengono in realtà ai programmi informatici assai più che non al lavoro nostro e dei nostri colleghi!).

Tito Orlandi - a cui sono debitore per grande parte di questa mia impostazione - nota anzi nel suo recente Informatica testuale una sorta di paradosso: la macchina informatica è talmente potente e duttile da poter "mimare" i media precedenti (nel nostro caso la stampa e la tipografia), ma questo stesso vantaggio pratico si tramuta in un ostacolo teorico, giacché noi siamo portati a credere che scrivendo con un computer noi produciamo ancora delle a oppure delle $\mathrm{b}$ analogiche e non invece una serie di segni digitali. Ciò che accade effettivamente nel mondo del digitale è invece assai diverso, più complesso, e comunque infinitamente più ricco di potenzialità non meno che di problemi teorici che chiedono di essere affrontati.

\section{Delimitazione del campo}

Si apre dunque un autentico universo di problemi teorici, all'interno del quale occorre scegliere drasticamente. Non foss'altro che per cercare di rispettare i limiti del mio intervento, non parlerò qui del problema che più mi sta a cuore, a cui ho dedicato uno specifico lavoro (Mordenti, Parádosis. A proposito del testo informatico), cioè del problema della persistenza del senso del testo e di perché dalla modalità informatica del testo non possano e non debbano affatto derivare le teorie della "deriva ermetica" e (in ultima analisi) della insignificanza del testo. La questione è davvero fondamentale perché, come scrive Francesco Fiorentino in un bel saggio che apre il volume da lui recentemente curato: "Questo caos [...] dopo essere stato un medium di liberazione (anche di nuova conoscenza) diventa un problema" ("Infinite reti: la letteratura nell'ipertesto della cultura" 56).

Il mio intervento presenta invece un'esperienza di edizione critica digitale che cerca di svolgersi prendendo sul serio l’informatica, cioè non solo utilizzando le sue "facilitazioni," ma cercando di sperimentare le sue potenzialità. Tali potenzialità richiedono, proprio per poter essere utilizzate a pieno, una complessiva riconfigurazione del lavoro filologico. In sostanza, il mio saggio parla dei problemi che ho incontrato e incontro nel lavoro volto a stabilire un'edizione critica informatizzata dello Zibaldone Laurenziano, manoscritto autografo di Giovanni Boccaccio, conservato alla Biblioteca Laurenziana di Firenze (Pluteo XXIX, 8), piuttosto che presentare lo stato (sempre iniziale e sperimentale) di questa edizione, ${ }^{1}$ giacché sono convinto che viviamo una fase in cui è più produttivo discutere dei problemi aperti che non illustrare i risultati già portati a compimento (che tuttavia ormai non mancano).

Vorrei prevenire un'obiezione legittima, anzi consueta: si potrebbe dire che concentrarsi sui problemi ancora aperti, piuttosto che sui risultati già conseguiti, somiglia un po' troppo al gesto della volpe che non considera mai abbastanza matura l'uva che non può 
raggiungere. C’è del vero in questa obiezione, e io stesso cito spesso l'affermazione del grande filologo Gottfried Hermann (nata dallo spirito polemico per lo stanco riproporsi del dibattito sul metodo fra lachmanniani e anti-lachmanniani): "Wer nichts über die Sache versteht, schreibt über die Methode" ("Chi non capisce nulla della pratica scrive del metodo”). ${ }^{2}$ Eppure, a fronte della portata dell'innovazione prodotta dall'informatizzazione del testo, credo che ora si dovrebbe rovesciare quel motto e affermare: "Chi oggi non scrive del metodo non può capire nulla della pratica.”

\section{Qualcosa a proposito dello Zibaldone Laurenziano e delle ragioni del suo interesse}

Mi limito a dire dello Zibaldone Laurenziano di Boccaccio, solo ciò che motiva il suo straordinario interesse e, brevemente, ciò ci permetterà di esplicitare un abbozzo di ragionamento a proposito delle caratteristiche dell'edizione informatizzata. ${ }^{3}$ Quanto ai motivi di interesse filologico di questo autografo boccacciano, basterà dire che esso contiene (fra molte altre cose, comprese alcune lettere petrarchesche) anche la pressoché unica attestazione dello scambio di egloghe fra Dante e Giovanni del Virgilio; e quanto alla sua importanza per la storia della cultura e della letteratura italiana, sarà sufficiente ricordare che questo manoscritto rappresenta il principale strumento per comprendere la formazione e l'evoluzione letteraria di Boccaccio (Branca "Giovanni Boccaccio. Profilo biografico," 40segg.).

Il libro-zibaldone medievale, un modello destinato a durare ancora a lungo nell'assetto culturale di ancien régime (dunque fino al giovane Leopardi), rappresenta un "libro-archivio d'autore," un “'libro d'autore' con la funzione d'un ms-archivio con la funzione d'un msarchivio" come Armando Petrucci definisce la forma-zibaldone (Petrucci, "Minuta, autografo, libro d'autore" 403; Bartoli Langeli, Infelise 444-55). Si tratta di un collettore di testi esemplari e/o utili che l'autore accumulava e conservava presso di sé, anche in vista di un successivo ri-uso, e si comprende bene l'importanza di un tale strumento in un periodo storico di scarsa diffusione, di difficile accessibilità e di alto costo dei libri. I testi che Boccaccio accumula in questo suo "libro-archivio d'autore" si arricchiscono continuamente, in pratica lungo tutta il corso della sua vita. Inoltre questi testi vennero sottoposti da Boccaccio a un continuo lavorìo di rielaborazione e riuso, essendo essi destinati a partecipare agli sviluppi ulteriori della sua attività letteraria (proprio a questo serviva infatti il "libro-archivio d'autore"). Se lo si legge in questa prospettiva, lo ZL presenta dunque un doppio processo intertestuale di straordinario interesse.

In primo luogo, attraverso le sue pagine diventa leggibile l'intero percorso della originale formazione culturale e letteraria di Boccaccio, e si percepisce perfettamente la vera linea di frattura che l'attraversa, simboleggiata dalla differenza e dall'antitesi fra le tre diverse parti di questo manoscritto: dalle lezioni di astronomia medievale di Andalò del Negro (dei ff. 225) e dalle pagine (ancora cronologicamente precedenti, cioè esemplate da un Boccaccio giovanissimo o addirittura adolescente: ff. 26-45) di vite memorabili ed esemplari, di storie antiche e leggendarie ecc., fino all'instaurarsi del nascente modello petrarchesco, il ricordo dell'incoronazione poetica di Petrarca (f. 73r) e le sue epistole già umanistiche ricopiate da 
Boccaccio nelle ultime pagine (ff.73r-74v, 76r ecc.). Si legge insomma sulle pagine di uno stesso manoscritto un passaggio di fase (o di epoca) che si verifica attraversando la variatissima cultura della Napoli angioina ${ }^{4}$ e, naturalmente, la lezione dell'altro modello possibile (e in Boccaccio ancora e sempre confliggente con quello petrarchesco): Dante Alighieri. Si tratta delle raccolte di storie e detti memorabili, delle allegorie, dei mirabilia, dei versi latini e delle egloghe, di frammenti epigrafici e di alfabeti greco ed ebraico ricopiati; e ancora: le lettere e le prime prove poetiche latine dello stesso Boccaccio (f.60r-v), il citato scambio di Egloghe fra Giovanni del Virgilio e Dante, e anche le lettere di Dante (ai ff. 62v-63r) ecc., che si leggono nelle parti centrali dello stesso codice. Questo (come attesta Quaglio) è:

Vivace di multipli interessi culturali e fervido di tradizioni antiche e più recenti: che si giustappongono in un coro discorde di voci..., incline com'è ad accogliere esigenze contrastanti, a contaminare pacificamente sacro e profano, scienza e letteratura, eredità sveva e gusti francesi. (Quaglio 144)

Una estrema varietà di testi, dunque, ma una varietà tutt'altro che casuale o insensata (tutt'altro che "zibaldonesca" nel senso contemporaneo del termine), dato che si costruisce con assoluta consapevole intenzionalità e si muove lungo una linea evolutiva di straordinaria portata ed evidenza.

In secondo luogo, si trova in queste pagine un processo intertestuale, o più precisamente intratestuale, che lega i diversi testi che Boccaccio ha selezionato e conservato nello ZL con quelli di cui egli è Autore. Consideriamo ad esempio il tema della misoginia, che compare nella pagina 52v dello ZL con il Contra Iovinianum (o De non ducenda uxore) attribuito a San Girolamo, e che deriva a sua volta da un testo pseudo-teofrasteo, il De Nuptiis (Migne 276-78). Proprio questo testo sarà in seguito riutilizzato costantemente da Boccaccio (e talvolta tradotto in lingua volgare ad verbum) in diverse sue opere: nel Trattatello in laude di Dante, naturalmente nel Corbaccio, e poi nelle ultime Esposizioni sopra la Comedìa (XVI, 28-45), dove si commenta il destino dei tre sodomiti fiorentini e le parole di Iacopo Rusticucci: "la fiera moglie più ch’altro mi noce."”

\section{Alcuni problemi irrisolti}

Come disse Claude Cazalé Bérard nella sua relazione al citato e memorabile seminario fiorentino sugli zibaldoni del 1996, lo ZL rappresenta anche: "un coacervo di problemi irrisolti.” Quali sono questi problemi? Tralascio qui non solo il problema dell'autografia dell'intero manoscritto (che impegnò in passato i filologi e che deve essere data ormai per provata), ${ }^{6}$ ma anche quelli brillantemente risolti nel frattempo (soprattutto da Stefano Zamponi e dal suo gruppo di ricerca, su base codicologica e paleografica). ${ }^{7}$ Basterà qui dire che anche dopo la conclusione della formidabile impresa dell'edizione di Tutte le Opere di Boccaccio diretta da Branca, alcuni testi presenti nello ZL sono ancora inediti (ad eccezione, evidentemente, di quelli di cui Boccaccio è autore). Come si spiega questo fatto? Basta considerare le regole consolidate della filologia dei secoli XIX e XX (pre-informatica, dunque): ricordiamo che Boccaccio nello ZL è il copista, non l'autore, e dunque anche nei 
casi in cui i testi presenti nello ZL sono stati presi in esame per altre edizioni critiche (ad esempio da parte di Ezio Franceschini per la sua edizione del Liber Philosophorum) essi figurano tutt'al più in stemmata codicum molto ricchi e complessi e non sempre nei rami più alti di tali stemmata.

Se è vero che questo imponevano le regole della filologia del tempo, è anche vero che queste regole non sembrano più sufficienti, perché noi vorremmo (e forse dovremmo) saperne di più sui testi di cui Boccaccio s’è appropriato ricopiandoli nel suo repertoriozibaldone. Così come saremmo interessati a sapere, se possibile, a partire da quali testi Boccaccio copiava, e attraverso quali intermediari, perché le filiere della tradizione non sono indifferenziate e il loro significato non è affatto identico. E ancora: esiste, oppure no, anche un legame di tipo sintagmatico fra i testi riuniti nello ZL? E se (o quando) un tale legame esiste, è allora possibile pubblicare i diversi segmenti testuali dello ZL separatamente, uno per uno (rinviando di volta in volta ad Aristotele ciò che appartiene ad Aristotele e a Petrarca ciò che è di Petrarca, ecc.), senza provocare in tal modo una perdita di informazione e di senso del nostro ZL?

Ma soprattutto noi non possiamo oggi non porci la questione seguente: è davvero così netta, nel Medio Evo della mouvance e della polifonia (Zumthor; Cerquiglini), la distinzione oggi vigente fra il copista e l'autore? Intendo dire che noi vorremmo sapere come Boccaccio interpretava-copiando (o copiava-interpretando) i suoi antigrafi, insomma come leggeva, in che modo e in quale misura comprendeva ciò che veniva copiando, come (per ipotesi) si sbagliava leggendo e/o modificava scrivendo, come incrementava quei testi o ne sopprimeva parti, come li innovava o li deformava, in che modo, e in quale misura, egli s'appropriava di quei testi che - con un solo e medesimo gesto, unitario e non separabile - egli leggevamemorizzava-scriveva-copiava-modificava.

In realtà, se noi guardiamo lo ZL attenti a ciò che Armando Petrucci definisce la “relazione di scrittura”, noi vediamo che Boccaccio riprende e pratica tutte e quattro le modalità faciendi libros definite da San Bonaventura:

(1) il modo dello scriptor, colui che "scribit aliena, nihil addendo vel mutando";

(2) quello del compilator, colui che "scribit aliena, addendo, sed non de suo";

(3) quello del commentator, il quale "scribit et aliena et sua" (ma restando ancora il suo apporto secondario e subordinato in rapporto al testo altrui);

(4) e infine il modo dell'auctor, il quale "scribit et sua et aliena, sed sua tanquam principalia”, e solo quest'ultimo tipo, precisa Bonaventura, "debet dici auctor" . (Faccio notare timidamente che una ipotetica quinta modalità, quella che per noi oggi descriverebbe un Autore, cioè colui che «solum scribit sua et non aliena», non è affatto contemplata da Bonaventura, il quale in realtà non poteva neppure immaginarne l'esistenza.)

Ora, non solamente queste quattro modalità dello scrivere coesistono nello ZL, ma esse si combinano e si contaminano inevitabilmente fra loro, e i loro confini si confondono di continuo. Il problema che emerge è dunque il seguente: si può applicare anche a questo testo medievale, nonostante la sua costitutiva mobilità, la nostra rigida "griglia 
dell'identificazione," secondo la quale a ogni testo corrisponderebbe sempre un autore e uno solo, un titolo e uno solo, e un solo stato di quel testo ne varietur? Ebbene, sembra che qualcosa di vitale del nostro testo medievale rischierebbe in tal modo di essere contraddetto e di andare definitivamente perduto nell'edizione.

Il fatto è che (forse ce ne accorgiamo solo adesso) quella griglia dell'identificazione (che la filologia pre-informatica ha adottato e anzi posto a suo fondamento) era in realtà una griglia gutemberghiana, cioè molto legata all'epistemologia (prima ancora che alla tecnologia) della stampa (Mordenti, La conoscenza in rete come bene comune). E se ne comprende bene la ragione: il testo di Gutenberg è destinato a essere validato in modo straordinario, per mezzo di una riproduzione che lo moltiplica sempre identico a se stesso (la stampa aspira a creare, al limite, un numero infinito di copie tutte identiche, cioè essa moltiplica nell' identità), allora si capisce che un tale testo debba essere pensato come "il Testo," il solo testo possibile e necessario, con l'articolo determinativo e l'iniziale maiuscola. Ma domandiamoci: era già così per il testo manoscritto pre-gutemberghiano? Ed è ancora così per il testo informatico post-gutemberghiano?

\section{Il problema del movimento testuale e le possibilità dell'edizione informatizzata}

A me sembra che il testo medievale (e ancor più un testo del tipo-zibaldone) presenti delle caratteristiche intrinseche che entrano in contraddizione con l'immobilità e la natura definitiva tipiche del testo a stampa; peraltro tali caratteristiche: a) non appartengono affatto al testo manoscritto in quanto tale, bensì al suo essere per la stampa; b) non appartengono affatto alle modalità informatiche dell'edizione.

La prima di queste caratteristiche, è il movimento, testuale, intertestuale, intratestuale: lo zibaldone in particolare è in effetti essenzialmente un testo in movimento, giacché esso è un testo che deriva da altri testi e che è destinato a produrre altri testi, o a mescolarsi ad essi. Ma proprio il movimento, lo sappiamo bene, rappresenta orrore e scandalo per l'epistemologia di Gutenberg e quindi dobbiamo ribadire che è l'epistemologia di Gutenberg che ci deve interessare, e non tanto la sua tecnologia. D’altra parte il movimento del testo rappresenta anche il limite invalicabile per la filologia che si è determinata anche a partire da Gutenberg: la possibile inesistenza di un archetipo, la contaminazione, le situazioni di recensio aperta, le varianti d'autore, non sono forse questi altrettanti hic sunt leones, del tutto insuperabili, per tale filologia? E, come è noto, proprio l'opposizione a tale pretesa immobilità del testo, assunta come postulato dalla filologia tradizionale, è ciò che motiva la “critica genetica” di matrice francese, e ne costituisce la rilevante verità interna (Grésillon; Giaveri-Grésillon).

A ben vedere, il sistema delle note a piè di pagina o le tavole delle lezioni alternative rifiutate o altri dispositivi tipografici tesi a dar conto del variare del testo, spesso inutilizzabili e comunque di norma inutilizzati, rappresentano esattamente il luogo dove si rivela la contraddizione insolubile fra il movimento proprio del testo e la rigidità propria della stampa. Ma il movimento del testo rappresenta per noi un concetto-chiave, almeno 
quando (come nel nostro caso) l'attenzione si sposta dal prodotto finale (ammettendo che questo possa esistere) al processo storico che presiede alla scrittura del testo, nel tempo e attraverso la tradizione o, come preferisco dire, attraverso la paràdosis.

Domandiamoci: questa impossibilità di rendere conto nell'edizione del movimento testuale vale ancora nel caso di una edizione informatizzata ${ }^{9}$ La risposta a questa domanda è no; l'edizione informatizzata, quella che si può basare sull'ipertestualità, sull'ipermedialità e sul ricorso alla rete, quella che Jean-Louis Lebrave ha definito come "l'édition critique au XXI.e siècle" (Lebrave 127), è perfettamente in grado di rendere nell'edizione il movimento testuale, restituendo al testo la sua rigorosa mobilità.

\section{II modello dell'edizione come ipertesto}

Considerata sotto questo aspetto e a partire da questo punto di vista, l'edizione informatizzata dello ZL a cui stiamo lavorando ci appare allora in una forma decisamente diversa da quella tradizionale, e più precisamente si presenta come un ipertesto (cfr. Figura 1):

$\underline{\text { Figura 1: }}$ Modello dell'edizione informatizzata dello ZL come ipertesto ${ }^{10}$

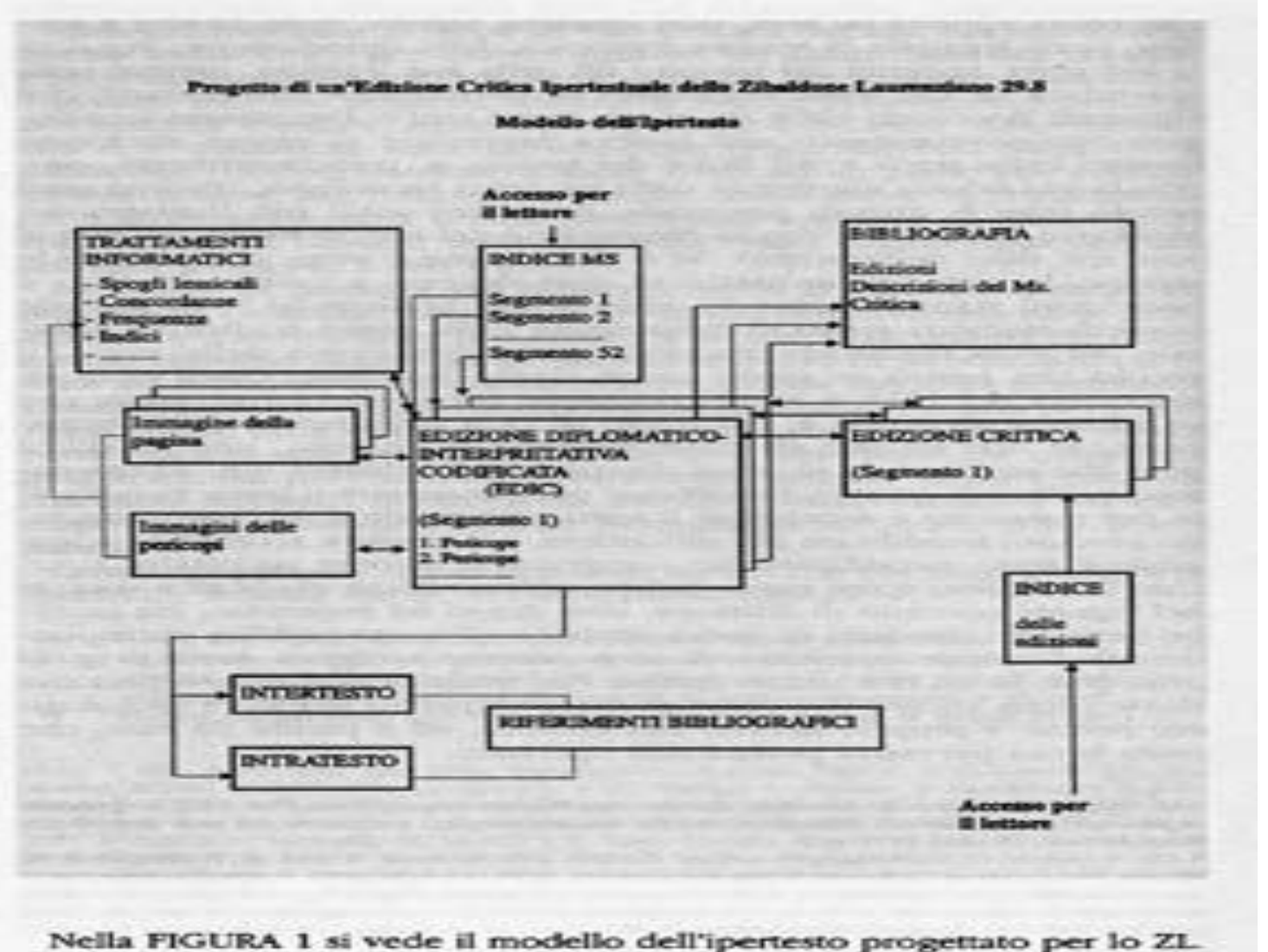

Presento due considerazioni a commento di questo modello:

1) È evidente dalla Figura 1 che il nostro lavoro di edizione si costituisce a partire da un insieme di edizioni (ove già disponibili), una per ogni segmento testuale ${ }^{11}$ che compone lo 
ZL; ciò significa che la nostra edizione sarà plurale e ipertestuale, cioè consisterà in una serie di ipertesti, dotati di un sistema ragionato di link capaci di legare istantaneamente, continuamente e utilmente ogni segmento non solo all'immagine del manoscritto e alla sua trascrizione codificata (su cui torneremo fra poco) ma anche al suo intertesto (i testi dai quali proviene o ai quali rinvia) e al suo intratesto (in particolare: i testi dello stesso Boccaccio che lo utilizzano o ne dipendono), rinviando naturalmente anche alla bibliografia critica specifica. Qui il plurale (“edizioni”) è anche teoricamente molto importante: recensendo nel 1946 un’opera di Boccaccio, l'Amorosa visione, edita nel 1944 da un giovane Vittore Branca, Gianfranco Contini si domandava, a proposito "del testo della princeps, dichiarato seconda redazione d'autore” : perché non stamparla, come sarebbe stato tanto più comodo, a fianco della prima? (Contini 555). La risposta che ci permettiamo di dare a questa domanda di Contini è forse un po' corriva ma sostanziale: perché il testo dell'Amorosa visione, nella graziosa edizione non critica, ottocentesca e tascabile, che mi ritrovo in casa (Amorosa visione composta per messer G. Boccaccio), consta di 50 canti e di ben 174 pagine. Questa risposta non vale più, evidentemente, nel caso di un'edizione informatica e on line.

2) Al centro del nostro sistema ipertestuale si trovano (come si vede ancora nella Figura 1) delle edizioni diplomatico-interpretative codificate, in sigla EDIC, cioè delle trascrizioni per la macchina informatica che sono necessarie affinché questa possa applicare tutta la sua potenza ordinatrice; occorre sottolineare (come è evidente nella nostra Figura 1) che tutti i trattamenti informatici ulteriori che la nostra edizione vuole permettere (spogli elettronici, concordanze, frequenze, analisi semantiche, ecc.) derivano e debbono derivare da una tale EDIC, e non dall'eventuale edizione cartacea, anche se questa fosse digitalizzata; così appare chiaramente che è proprio in questa fase della trascrizione (e più precisamente nel lavoro di codifica e di mark-up di cui consiste l'EDIC) che consiste il vero valore aggiunto dell'edizione informatizzata, ciò che la differenza nettamente da qualsiasi edizione cartacea gutemberghiana.

Insomma, l'edizione informatizzata è - e deve essere - un moltiplicatore di conoscenze ulteriori, ed è per questo che abbiamo proposto di definirla una "edizione sapiente;" si tratta evidentemente di un calco dell'espressione francese "édition savante" (usato normalmente in francese per dire dell'edizione critica), ma a cui vorremmo attribuire un senso più profondo, cioè significare che una edizione critica informatizzata deve essere pensata e costruita in modo tale che essa possa essere utilizzata in vista di trattamenti ulteriori: trattamenti analitici svolti con l'aiuto della macchina informatica, per rispondere alle esigenze di altre discipline, quelle filologiche e critico-letterarie, ma anche quelle linguistiche, paleografiche, storiche ecc., e perfino (o soprattutto?) quelle future di una natura tale che noi oggi non possiamo neppure immaginare.

\section{II momento centrale: la codifica}

Affinché tutto questo possa realizzarsi, è però necessario che l'edizione informatica sappia porsi, e risolvere nel migliore dei modi, il problema della codifica (che appare dunque come assolutamente cruciale per l'intera nostra operazione ecdotica); la 
trascrizione, intesa come codifica, è davvero il cuore dell'edizione informatica, mentre essa era un momento assolutamente secondario e, al limite, pre-scientifico, nella procedura ecdotica gutemberghiana. $^{12}$

Il problema di tale codifica è che essa si deve svolgere assumendo, in un certo senso, la macchina informatica come proprio destinatario, e ciò significa che essa deve svolgersi secondo i principi di esplicitezza, di rigore, di univocità, di non ambiguità e di non ridondanza, che la macchina stessa richiede per poter funzionare; non si può invece ricorrere, in alcun modo, a quelle che D'Arco Silvio Avalle "definiva le bonarie tassonomie semi-clandestine" (Avalle 380) che caratterizzano la comunicazione fra umani, al "più o meno è uguale" e al "tanto ci capiamo..." che noi usiamo abitualmente.

Il testo così codificato in SGML-XML si presenta nel modo che si può vedere qui di seguito (nella Figura 2), per meglio dire esso viene offerto in questo modo dal nostro lavoro umano e manuale alla capacità di elaborazione della macchina informatica.

Figura 2: Una pagina dello ZL (f. 31v, righe 1-19) codificata per la macchina

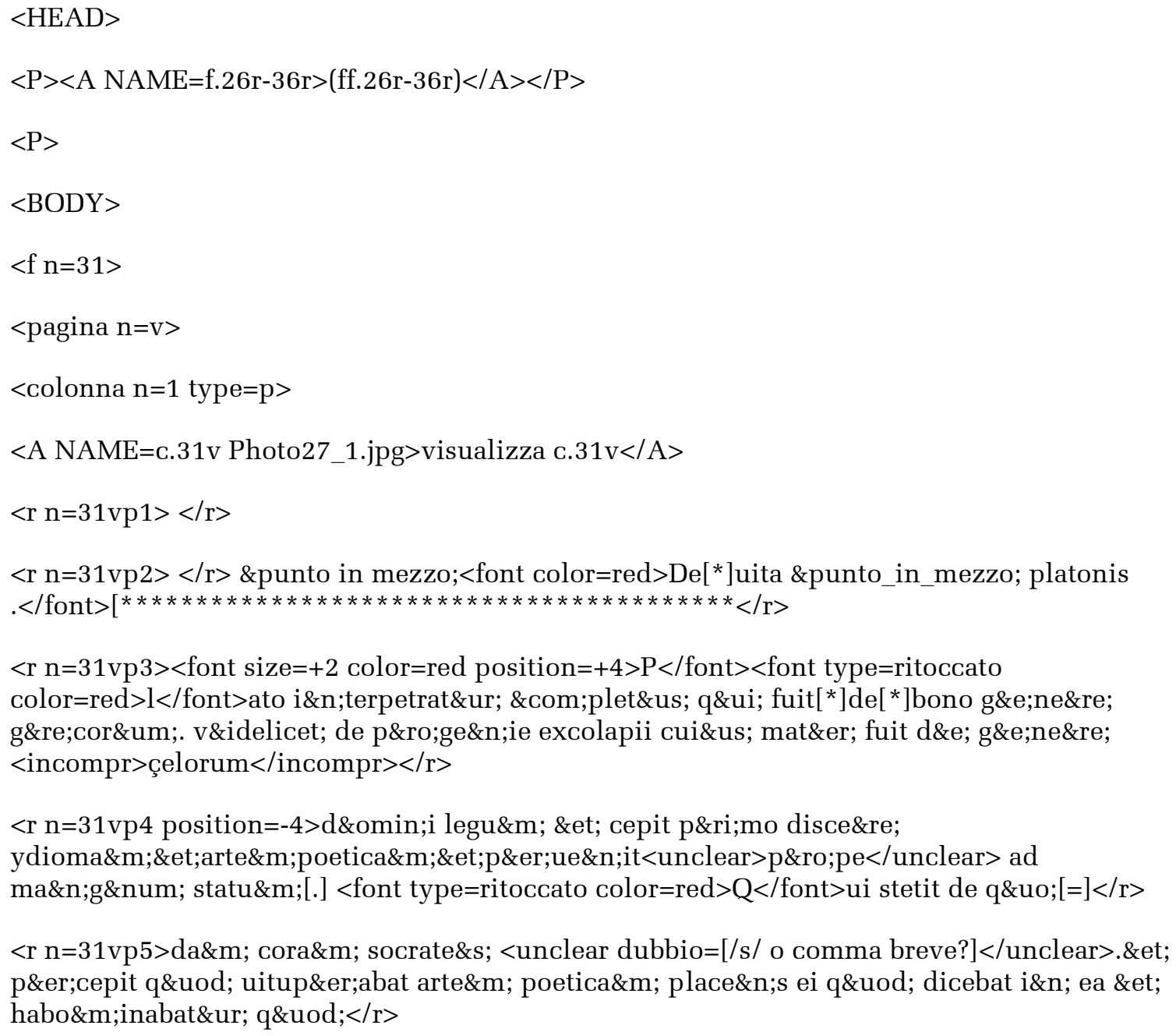


$<\mathrm{r} n=31 \mathrm{vp6}>$ sciebat i\&n; ea no\&n; recedens a \&punto_in_mezzo; $<$ font type=ritoccato color=red $>S</$ font $>$ o\&crate; \&punto_in_mezzo; p\&ri;\&us; q\&uam; audiret eu\&m; \&punto_in_mezzo; v \&punto_in_mezzo; anni\&s; \&et;

obeunte\&punto_in_mezzo; $<$ fonttype $=$ ritoccatocolor $=$ red $>S</$ font $>$ o\&crati;\&punto_in_mezzo; dixeru\&n;t plato\&n;i q\&uod; $</ r>$

$<\mathrm{r} n=31 v p 7>$ in [*] egipto era\&n;t aliqui pitagore discipuli ad q\&uo;s accessit \&et; p\&ro;ficiens cu\&m; eis rediit ab egipto $</ r>$

$<\mathrm{rn}=31 \mathrm{vp} 8><$ unclear dubbio=[rivedere] $>$ aethenas $<$ /unclear $>$ u\&bi; statuit duas scolasin[*]sci\&ent;ia duce\&n;s laudabiliore\&m; vita\&m; <dubbio>q\&uam;</dubbio $>$ possibil\&is; e\&ss; et i\&n; faciendis $</>$

$<\mathrm{r} n=31 v p 9>$ pulcris op\&e;ib\&us; in[*]fouendi\&s; egenis nitente\&s; aut\&em; ut eis d\&omi;naret\&ur; n\&ec; voluit q\&uia; recep\&er;it eos ma[=]

$<$ r n=31vp10>le ordinatos \&et; q\&uia; a\&n;i\&m;a\&l;b\&us; eo\&rum; p\&re;ualuera\&n;t aliq\&ui; more\&s; quos i\&n; ei\&s; p\&er;mutare no\&n; \&con;fidebat $<$ font type=ritoccato color $=$ red $>$ E $</$ font $>$ t si for $[=]</ r>$

$<\mathrm{r} n=31 \mathrm{vp} 11>$ te conaret\&ur; eos p\&er; $\left[{ }^{\circ}\right]$ mutare $\mathrm{ab}\left[{ }^{*}\right]$ ill\&is; occidere\&n;t illu\&m; sic\&ut; $<$ incompr $>$ ea $<$ incompr $>$ mag\&ist;r\&u;m \&punto_in_mezzo; $<$ font type=ritoccato color $=$ red $>$ S $</$ font $>$ o\&cratem; \&punto_in_mezzo; occideru\&n;t $<$ font type=ritoccato color $=$ red $>$ E $</$ font $>$ t vix\&it; \&punto_in_mezzo;lxi\&punto_in_mezzo; $</ r>$

$<\mathrm{r} \mathrm{n=31vp12>anno} \mathrm{\& et;} \mathrm{fuit} \mathrm{bo \& n;a \& rum;} \mathrm{disposit \& i;onu \& m;} \mathrm{\& et;} \mathrm{mo \& rum;} \mathrm{collator}$ bo\&no;\&rum; co\&n;sanguineis \&et; ex\&tra;nei\&s; \&et; multe patien[=] $</ r>$

$<$ r n=31vp13>tie \&et; h\&ab;uit multos discipulos post cui\&us; morte\&m; suns\&er;u\&n;t scolas eo\&rum; duo un\&us; d\&icitu;r\&punto_in_mezzo; cazenocra[=]

$<\mathrm{r}$ n=31vp14>te\&s; \&punto_in_mezzo; \&et; alt\&er_finale; aristotile\&s; <font type=ritoccato color $=$ red $>E</$ font $>$ t o\&ste;ndit seu dix\&it; p\&er; allegoria\&m; sua\&m; sc\&ent;iam ocultans $<$ correz=riscrittura $>$ ea\&m; $<$ correz $>$ ut ea\&m; no\&n; intellige\&ren;t $</ r>$

$<\mathrm{r}$ n=31vp15 $>$ n\&isi; sapiens $<$ font type=ritoccato color=red $>$ E $<$ ffont $>$ t dicit $\mathrm{a}<$ correz=riscrittura $>$ ty $</$ correz $>$ meo \&et; socrate assu\&m;me\&n;s [sic] ab ei\&s; plure\&s; opp\&osit\&io\&n;e\&s; ex quib\&us; \&com;posuit \&punto_in_mezzo;lvj\&punto_in_mezzo; lib\&r; $<$ posiz=interlineo $>$ os $</$ posiz $></$ r $>$

$<\mathrm{r} n=31 \mathrm{vp} 16><$ font type=ritoccato color $=$ red $>\mathrm{Q}</$ font $>$ ui fuit dimissi coloris pulcre for\&me; bo\&n;e disposit\&i;o\&n;is for ${ }^{\circ}$ mosior oc\&u;lor\&um; i\&n; barba <correz=riscrittura su macchia $>$ h\&abe;ns $<$ /correz $>$ sing\&num; unu\&m;</r $>$

$<\mathrm{r}$ n=31vp17>fuit s\&u;btiliu\&m; <correz >riscrittura $\mathrm{d} / \mathrm{v}<$ /correz $>$ v\&er;bor\&um; diligens semp\&er; secede\&re; ad des\&er;tu\&m; \&et; ut ingnorante\&s; e\&ss;ent \&propr;ij locis sui p\&er; uoce\&m; $</ r>$

$<\mathrm{r}$ n=31vp18>ip\&s;i\&us; qua\&m; exaltando alij audieba\&n;t q\&uas;i circa duo <unclear dubbio=[forse dopo "milia" un "adiebant"?] $>$ milia\&r;ia</unclear $>$ i\&n; locis i\&n; abitati\&s; \&punto_in_mezzo; $<$ font color=red $>$ Dicta \&punto_in_mezzo; plato\&n;is $</$ font $></$ r $>$

$<\mathrm{rn}=31 \mathrm{vp} 19><$ font $\operatorname{size}=+2$ color $=$ red position $=+4>\mathrm{E}</$ font $><$ font type=ritoccato color $=$ red $>\mathrm{T}</$ font $>$ p\&re; dicauit... 
Mi rendo ben conto che la Figura 2 non offre certo una bella pagina per la lettura di noi umani, senza contare il problema ben più rilevante del tempo di lavoro necessario per produrre una simile codifica, che per giunta è da considerarsi ancora e in una certa misura sempre provvisoria. Ma ne vale la pena. Per concludere cerchiamo, anche in questo caso con un solo esempio, di motivare questa ultima affermazione.

\section{Noi nani issati sulle spalle di giganti}

L'esempio che voglio allegare riguarda la possibilità (su cui sto lavorando proprio in questi mesi) di utilizzare l'informatica per descrivere l'evolvere nel tempo della scrittura di Boccaccio (o meglio: di alcune sue varianti glifiche) e, per questa via, di trarre elementi utili ai fini di una datazione più precisa e attendibile dei testi.

Nella sua descrizione dello ZL del 1975, Di Benedetto rilevava losconcertante alternarsi - oltre che di inchiostri - di tipi di scrittura varii per modulo, per disegno, per ritmo: quasi un campionario delle scritture usate da Boccaccio nel lungo arco della sua attività di copista ${ }^{13}$. (Di Benedetto, "Lo Zibaldone Laurenziano, libro segreto del Boccaccio” 120)

Ora proprio una tale varietas della scrittura di Boccaccio può offrire un contributo prezioso per la soluzione di un problema classico e illustre della nostra filologia, quello della datazione delle opere di Boccaccio, in questo caso dei diversi segmenti presenti nello ZL.

In effetti, per far questo, noi possiamo oramai poggiarci, come su un acquisto metodologico fondamentale, sulla ricerca di Vittore Branca e di Pier Giorgio Ricci del 1962, a proposito della storia della mano di Boccaccio, da loro tracciata in base all'analisi di certi tratti grafici (o meglio glifici) in evoluzione lungo il tempo (Branca, Ricci). Come è noto, quella ricerca - occasionata dal dibattito intorno all'autografia del Decameron Berlinese del cod. Hamilton 90 - poté rovesciare un deficit di conoscenza (cioè il mancato riconoscimento della mano di Boccaccio, a causa delle forti differenze della scrittura del Berlinese rispetto ad altri codici attribuiti con certezza) nel suo contrario, cioè in un incremento preziosissimo di informazioni a proposito della datazione. A partire dalla concezione dell'autografia di Boccaccio come un dato variabile nel corso del tempo, secondo precise scansioni testimoniate da altri autografi datati, derivò in effetti non solo un contributo decisivo per l'attribuzione alla mano di Boccaccio del Berlinese ma anche la possibilità di datare con relativa precisione quel testo intorno al 1370, dunque di mano di un Boccaccio ormai vecchio.

Ma questa operazione di datazione sulla base dell'evoluzione del grafismo e dell'usus scribendi di un autore riprendeva a sua volta un insegnamento ancora più antico, in particolare quello di uno dei padri fondatori della filologia italiana, Michele Barbi, che aveva potuto utilizzare questo medesimo metodo con assoluta naturalezza. Si tratta in questo caso di una conoscenza autentica e profonda, che non si può certo confondere con la magia o la divinazione, ma che rese possibile un celebre gesto di Barbi, poi trasmesso dai 
suoi allievi quasi come una leggenda: messo finalmente di fronte nella Biblioteca Laurenziana al dibattutissimo codice Berlinese Hamilton 90, Barbi lo prese in mano, lo sfogliò, ne lesse alcune parti, e poi proclamò con assoluta certezza: "È suo. E degli ultimi anni.” Insomma in pochi istanti Michele Barbi capì quello che solo decenni dopo sarebbe stato compreso, e tanto faticosamente dimostrato, non solo riconoscendo l'autografia del Decameron Berlinese ma anche collocandolo con perfezione nel tempo della vita di Boccaccio. $^{14}$

Ma come poté Barbi far questo? Certamente perché era accumulato in lui uno straordinario sapere analitico a proposito della grafia di Boccaccio e della sua evoluzione nel tempo, e ciò di certo grazie alla sua eccezionale conoscenza diretta dei manoscritti di Boccaccio, verosimilmente soprattutto di Boccaccio in quanto assiduo copista di testi danteschi. Alla recensio sistematica di tali testi Barbi era infatti impegnato fin da giovane, prima per la sua edizione critica della Vita Nuova poi per il lavoro della Società Dantesca, da lui diretto, rivolto all'edizione critica della Divina Commedia. Così Pier Giorgio Ricci descrive l'innovazione barbiana:

Nessuno, all'infuori di Barbi, conobbe la storia della grafia del Boccaccio, la quale fu densissima di tappe importanti, secondo un'instancabile linea di sviluppo che va dagli anni giovanili agli estremi. (Branca, Ricci 51)

Questa conoscenza è espressa da Barbi in una pagina, variamente riproposta nel corso degli anni (Introduzione 1907, CLXXI; "Qual è la seconda redazione del Trattatello in laude di Dante?”; Studi su Giovanni Boccaccio; Introduzione 1932, CXVI). La domanda è: possiamo noi rendere esplicito e formale il sapere che in Barbi era implicito e quasi naturale? E possiamo rendere tale sapere discreto, cioè tradurlo in una serie di elementi separati, in una tabella di corrispondenze, in modo che il computer possa prenderli in esame, quantificarli, computarli e restituirceli in forma di informazioni attendibili?

Per questo abbiamo scelto anche noi di dare conto, in sede di codifica dello ZL, dei tratti grafici di Boccaccio in evoluzione, che sono stati fatti oggetto di uno specifico mark up che ne permetterà in seguito il trattamento automatico: si tratta anzitutto degli stessi elementi che Barbi, Ricci e Branca avevano già messo in evidenza (le varianti glifiche della lettera $a$, l'alternanza di $u / v$ maiuscole, i due tipi di $r$ minuscola ecc.), ma anche noi ne veniamo aggiungendo altri nuovi e diversi da quelli, man mano che avanziamo (con l'aiuto paleografico decisivo della dottoressa Alda Spotti) nel nostro lavoro di edizione diplomatico-interpretativa codificata dello ZL. Noi speriamo di poter pervenire così a tracciare - grazie al computer - un diagramma completo dell'evoluzione di tali fenomeni e ci attendiamo da questo qualche risposta significativa in ordine alla datazione dei segmenti testuali che compongono lo ZL, e forse anche in ordine alla loro attribuzione autoriale.

Insomma, noi siamo veramente dei nani issati sulle spalle dei giganti che ci hanno preceduto, ma siamo nani che hanno nelle loro mani una preziosa macchina informatica che i giganti non potevano avere; ciò che ci permetterà, forse, di esaminare con precisione, di quantificare, di situare in modo analitico, d'interrogare dinamicamente ecc. proprio quei 
tratti della scrittura di Boccaccio che Barbi e quelli come lui sapevano valutare con un colpo d'occhio, solo sulla base del loro straordinario iudicium.

Conservando tutte le proporzioni, mi piacerebbe se questi nani che noi siamo potessero rivendicare di fare lo stesso mestiere di quei giganti loro maestri, comprovando in tal modo la vitalità, nel nostro nuovo tempo, di una disciplina umanistica che chiamiamo filologia.

\section{Conclusioni problematiche: qualche domanda}

In conclusione credo di poter solo formulare alcune domande (che spero sensate), a cominciare da quella che riguarda il concetto stesso di edizione.

Formulerei la prima domanda in questi termini: nel concetto attualmente vigente di edizione quanto è davvero essenziale al concetto stesso e quanto invece è debitore della stampa, intendo dire dell'epistemologia di Gutenberg non solo della sua tecnologia? E ancora: quale rapporto c'è fra gli obiettivi e i metodi che si è data la filologia che conosciamo e la tecnologia/epistemologia di Gutenberg? Forse questo rapporto è meno indiretto e labile di quanto siamo abituati a pensare. ${ }^{15}$

Penso soprattutto a cinque caratteristiche ${ }^{16}$ dell'edizione critica che noi conosciamo, e che abbiamo tutti/e ancora in testa a comandarci (magari senza che noi stessi ce ne accorgiamo):

1) al concetto di definitività dell'edizione (ne varietur!), e alla nettissima distinzione fra lavori preparatori - che restano, et pour cause, inediti nella configurazione gutemberghiana - e l'edizione vera e propria, cioè definitiva, del testo;

2) all'idea, legata al primo punto, ma logicamente da distinguere, che il testo da editare sia, e sia sempre, e debba essere sempre, uno e uno solo;

3) alla netta gerarchia fra testo e apparato, cioè fra la soluzione prescelta dall'editore in base allo stato - per definizione sempre provvisorio - della ricerca filologica, e le lezioni rifiutate, confinate nelle note;

4) alla nettissima gerarchia di importanza scientifica fra l'edizione critica propriamente detta e l'edizione diplomatica, o diplomatico-interpretativa, o, nel nostro caso, EDIC;

5) alla esclusiva autorialità dell'editore ${ }^{17}$ - chiamiamola contraddittoriamente e forse provocatoriamente così -, cioè al carattere assolutamente individuale, e non collettivo del lavoro di edizione.

Ora, io credo che si possa avanzare almeno l'ipotesi - dunque è questa la domanda più radicale che mi rivolgo e rivolgo al Lettore - che una modalità propriamente informatica di edizione $^{18}$ metta radicalmente in crisi, uno per uno, tutti e cinque questi punti appena richiamati. Esaminiamoli partitamente:

1) al concetto di definitività dell'edizione (ne varietur), e alla nettissima distinzione fra l'edizione e i lavori preparatori, che restano inediti nella configurazione 
gutemberghiana, si oppone, mi sembra, la modalità informatica dell'edizione critica; qui una possibilità tecnologica che consiste nel correggere e modificare l'edizione senza dover sopportare quasi nessun costo, ben diversamente da quanto avveniva nella configurazione gutemberghiana, si aggiunge utilmente a una opportunità scientifica, che definirei quasi di deontologia della ricerca, consentita dall'informatica. Mi riferisco alla possibilità, in un certo senso, alla necessità, di rendere disponibile il lavoro in ogni momento del suo farsi, affinché la comunità scientifica possa intervenire e discutere e, ove necessario, correggere. Faccio notare che questa modalità, lungi dall'essere prova di approssimazione e trascuratezza, è invece già oggi largamente praticata dai nostri colleghi scienziati, e un settore avanzatissimo e certamente assai concorrenziale al suo interno, come quello della fisica delle particelle, comunica da anni solo mettendo in rete, nella forma di "Archivi” on line accessibili a tutti e sempre più aggiornati, spunti, ipotesi di ricerca, pre-print e quant'altro. È stata questa, ad esempio, la modalità di comunicazione della recente possibile scoperta, poi smentita, del neutrino più veloce della luce, meglio nota al grande pubblico come il tunnel dal CERN di Ginevra al laboratorio del Gran Sasso che l'ex Ministro Gelmini si è vantata di aver finanziato.

2) All'idea (legata al primo punto, ma logicamente da distinguere) che il testo da editare sia, e sia sempre, e debba essere sempre, uno e uno solo, si oppone - di nuovo - ciò che abbiamo definito come la "rigorosa mobilità" dell'edizione consentita ora dall'informatica. È particolarmente suggestivo richiamare a questo proposito l'idea pasqualiana che non vi sia stato sempre un archetipo e uno solo, ma archetipi in movimento, tradizioni tutte genuine e però differenziate fin dalla loro origine, per non dire del fenomeno della contaminazione. Non c’è dubbio che questa idea - finora solo una stimolante critica teorica al lachmannismo troppo schematico - sia oggi praticabile anche in sede di edizione, dato che diventa ora del tutto possibile produrre edizioni che tengano conto concretamente di questa mouvance testuale.

3) Alla netta gerarchia fra testo e apparato, cioè fra la soluzione prescelta dall'editore in base allo stato (per definizione sempre provvisorio) della ricerca filologica e le lezioni rifiutate, confinate nelle note, si oppone (anche in questo caso) la possibilità tecnologica consentita dall'edizione informatica di produrre ipertesti in cui le lezioni rifiutate possano, con un solo clic, "andare a testo", e viceversa; e allora, se si può fare, perché non farlo?

4) Alla consolidata gerarchia di importanza scientifica fra l'edizione critica propriamente detta e l'edizione diplomatica, o diplomatico-interpretativa, si oppone radicalmente quanto abbiamo detto (v. supra: specie nel paragrafo 6) a proposito della centralità della trascrizione intesa come codifica. Se l'edizione informatica ruota interamente attorno a una codifica ben fatta, ecco allora che diventa vero il paradosso enunciato tanto tempo fa dal paleografo Masai, cioè che “solo le edizioni diplomatiche sono definitive," e che sulla loro base si potranno 
poi costruire $n$ edizioni critiche, tutte revocabili in dubbio a fronte dell'emergere di nuovi dati. Da questo punto di vista occorre chiedersi se l'edizione, secondo il magistero di Contini, sia o non sia solo l'ipotesi più economica che dà conto dei dati disponibili? E occorre tenere conto del fatto che essa può e deve cambiare di fronte all'emergere di nuovi dati, o semplicemente di una loro migliore interpretazione.

5) Alla esclusiva autorialità dell'editore ${ }^{17}$, cioè al carattere assolutamente individuale, e non collettivo del lavoro di edizione si oppone, molto semplicemente, la realtà dei fatti. Si può e si deve ora immaginare un’edizione a cui collaborino più editori, sia nel senso più stretto e diretto (che ad essa possono apportare pareri e proposte diversi editori on line) sia nel senso più vasto, ma altrettanto importante teoricamente, che ad essa vengano linkate altre edizioni disponibili, siano queste le edizioni diverse dello stesso testo, oppure l'intertesto o l'intratesto a cui il testo da editare rinvia.

In conclusione, per entrare davvero nella nuova fase della filologia digitale, dobbiamo cominciare a pensare a: (i) un'edizione sempre in fieri, mai definitiva (esattamente come è nella natura della ricerca, di ogni ricerca); (ii) un'edizione che non ricostruisce necessariamente una e una sola versione, o stato, del testo, ma che può ripercorrerne e riprodurne, con la "rigorosa mobilità" di cui è capace la macchina informatica e che è del tutto impossibile alla stampa, il movimento del testo nella tradizione; (iii) un'edizione che lascia aperto il vitale rapporto fra testo e apparato, o fra diversi rami della tradizione, o fra diverse versioni varianti ecc.; (iv) un'edizione che concentra il peso del lavoro scientifico sulla codifica (ma dunque - per dir così - sulla forma moderna e informatica dell'edizione diplomatica); (v) infine un'edizione che ha più padri, o madri, e che non appartiene in esclusiva a nessuno, ma appartiene alla comunità scientifica in quanto tale, perché è frutto di una sorta di straordinario cervello collettivo che lavora insieme, collabora, attraverso i confini e attraverso i secoli.

\footnotetext{
${ }^{1}$ Lo stato di avanzamento di questo lavoro, mentre esso ancora si svolge, si può comunque consultare presso il sito del CISADU della "Sapienza" Università di Roma, all'indirizzo: <http://rmcisadu.let.uniroma1.it/boccaccio/Zibaldone\%Laurenziano\%2029 8.htm>.

${ }^{2}$ Ad esempio ho voluto collocare proprio questa frase a esergo del mio libro Informatica $e$ critica dei testi.

${ }^{3}$ D'ora in poi, sarà da noi designato con la sigla ZL. Non si può non ricordare tuttavia che a proposito dello ZL tutto cominciò, o ricominciò, nel 1996, e precisamente da un seminario internazionale sugli zibaldoni di Boccaccio (organizzato da Claude Cazalé Bérard, in collaborazione con altri ricercatori e sotto il patronato del rimpianto Vittore Branca). Cfr. Picone-Cazalé Bérard..

${ }^{4}$ Probabilmente decisiva fu la mediazione nei confronti dei testi di Petrarca svolta da Dionigi da Borgo san Sepolcro. Cfr. anche R. Sabbadini, Le scoperte dei codici latini e greci $n e$ ' secoli $X I V$ e $X V$.

${ }^{5}$ Questa pagina dello ZL è stata affrontata e pubblicata informaticamente (per la sua tesi di laurea magistrale) da quella che considero forse in assoluto la più brillante fra le mie allieve,
} 
la quale attualmente è impegnata in tutt'altro lavoro (in verità assai lontano dalla sua formazione e dalle sue capacità) grazie alla straordinaria efficacia classista del nostro sistema di reclutamento dei giovani ricercatori; cfr. Feliziani, Per l'edizione critica informatizzata dello Zibaldone Laurenziano,

${ }^{6}$ Cfr. Henri Hauvette, Notes sur des manuscrits autographes de Boccaccio à la Bibliotheque Laurentienne. In verità Hauvette ritenne autografa solo la seconda parte del ms., da cc. 45v a 77r (e solo questa fu infatti pubblicata in fac-simile dal Biagi nel 1915. Hauvette ebbe comunque il grande merito di richiamare l'attenzione su quel venerando codice, come più in generale sugli autografi boccacciani. Si veda anche la recensione di F. Novati alle scoperte di Hauvette. Il problema dell'autografia fu riaperto da Filippo Di Benedetto, cfr. Considerazioni sullo Zibaldone laurenziano del Boccaccio e Lo Zibaldone Laurenziano, libro segreto del Boccaccio (120). La questione fu poi affrontata ancora da Anna Maria Cesari, in Presentazione del Codice Laurenziano Plut. XXIX, 8. Per un'esaustiva e conclusiva messa a punto dei problemi paleografici e codicologici dello ZL (e del suo affine e gemello: il cod. Mediceo Laurenziano, Pluteo XXXIII, 31, la cosiddetta Miscellanea laurenziana) si veda Zamponi-Pantarotto-Tomiello, "Stratigrafia dello Zibaldone e della Miscellanea laurenziana,” in Picone-Cazalé Bérard, 181-243, in particolare 181-190.

${ }^{7}$ Cfr. Bianca Maria Da Rif, e ora: Stefano Zamponi, Genesi e metamorfosi del libro segreto di Boccaccio. Per il palinsesto della pergamena (che rivela la comune provenienza di ZL e della Miscellanea) cfr. Virginia Brown..

${ }^{8}$ Bonaventura da Bagnorea, Commentarius in I Librum Sententiarum, cit. in: A. Petrucci, Minuta, autografo, libro d'autore, cit. p.402.

9 Parlando di "edizione informatizzata" (o informatica) intendo una edizione che si costruisce interamente a partire dalle potenzialità euristiche proprie della macchina informatica; dunque questo concetto va distinto da quello di un'edizione che faccia semplicemente un qualche uso dell'informatica, ad esempio passando per uno scanner le edizioni gutemberghiane già disponibili; per quest'ultima modalità propongo di utilizzare il concetto e la definizione di "edizione digitale."

${ }^{10} \mathrm{Cfr} .<$ http://rmcisadu.let.uniroma1.it/boccaccio/modello.html>.

${ }^{11}$ Ricordiamo che tali segmenti sono 55 secondo la Tavola della Cesari (454-466) e sono invece 72 secondo Filippo Di Benedetto, Presenza di testi minori negli Zabaldoni (26-28); la discordanza, non essenziale, è dovuta soprattutto a una diversa valutazione di alcune brevi composizioni riportate da Boccaccio.

12 Sulla necessità di superare ogni troppo netta distinzione fra trascrizione ed edizione critica, rinvio a R. Mordenti, Informatica e critica dei testi. Petrucci ha proposto l'espressione "trascrizione critica", che mi sembra meriterebbe di entrare nel nostro lessico con pienezza di significato.

13 Inoltre (come Zamponi nelle opp. citt. ha definitivamente dimostrato) la cronologia della scrittura non corrisponde affatto alla topologia, cioè al succedersi delle scritture sulle carte di quel codice.

${ }^{14}$ Tutt'altra questione, che non è certo possibile affrontare qui, è capire perché Barbi non abbia mai dato annuncio scritto di una simile scoperta (un autografo del Decameron!), che fu riferita solo dopo la sua morte, nel dopoguerra, dal suo allievo Alberto Chiari, accolto peraltro da un unanime scetticismo.

${ }^{15}$ Sia detto con prudenza e solo in questa nota scritta piccola: le date stesse forse ce lo suggerirebbero, collocandosi la nascita della filologia moderna nella piena fioritura dell'Umanesimo, cioè nella seconda metà del XV secolo e all'inizio del XVI, dunque proprio in coincidenza con l'avvento dell'era gutemberghiana. È solo una coincidenza? 
${ }^{16}$ Questa parte conclusiva ripercorre in gran parte un mio intervento: Domande teoriche sul concetto di edizione (nel nome di Giuseppe Gigliozzi), ora in G. Crupi - F. Ciotti (a cura di), Dall'informatica umanistica alle culture digitali. Convegno di studi in memoria di Giuseppe Gigliozzi, Roma "Sapienza”-"Tor Vergata”, 27-28 ottobre 2011 (in corso di stampa).

${ }^{17}$ Superfluo dire che qui si intende qui editore come editor e non come publisher.

${ }^{18}$ Cfr. supra nota 22.

\section{Works Cited}

Barbi, Michele. Introduzione. Alighieri, Dante. Vita Nuova, Hoepli, Milano 1907. ICCLXXXVII; Id. "Qual è la seconda redazione del Trattatello in laude di Dante?" Miscellanea Storica della Valdelsa, XXI (1913). 101-41; Id. Studi su Giovanni Boccaccio. Castel Fiorentino: Società storica della Valdelsa, 1913; Id. Introduzione a D. Alighieri, Vita Nuova. Firenze: Bemporad, 1932. I- CCCIX. Print.

Bartoli Langeli Attilio, e Infelise, Mario. Il libro manoscritto e a stampa, in L'italiano nelle regioni. Lingua nazionale e identità regionali. Ed. Francesco, Bruni. Torino: Utet, 1992. 941-977. Print.

Biagi, Guido. Prefazione. Lo Zibaldone Boccaccesco Mediceo Laurenziano Plut. XXIX .8 riprodotto in facsimile a cura della $R$. Biblioteca Medicea Laurenziana con prefazione del prof. dott. Guido Biagi. Firenze: Olschki, 1915. 1-9. Print.

Boccaccio, Giovanni. Amorosa visione composta per messer G. Boccaccio. Testo di lingua. Firenze: Tipografia Fiorentina, 1826. Print.

Branca, Vittore. “Giovanni Boccaccio. Profilo biografico.” Boccaccio, Giovanni. Tutte le opere. Ed. Vittore Branca. Vol. I, Caccia di Diana. Filocolo, Milano: Mondadori, 1967. 1203. Print.

Branca, Vittore, e Ricci, Pier Giorgio. Un autografo del Decameron (Codice Hamilton 90), Padova: CEDAM, 1962. Print.

Brown, Virginia. "Boccaccio in Naples: The Beneventan liturgical palimpsest of the Laurentian autographs (Mss.29. 8 and 33. 31)." Italia medievale e umanistica, XXXIV (1991): 41-126. Print.

Cerquiglini, Bernard. Éloge de la variante. Paris: Seuil, 1989. Print.

Cesari, Anna Maria. Presentazione del Codice Laurenziano Plut. XXIX, 8. Archivio Storico Lombardo, serie IX, anni XCVIII-XCIX-C (1971-1972-1973), vol.X, fasc.I-III: 434-477. Print.

Contini, Gianfranco. “L”Amorosa visione' nell'edizione di Vittore Branca” (1946), Frammenti di filologia romanza. Firenze: Edizioni del Galluzzo, 2007, vol. I, 555-590. Print.

Da Rif, Bianca Maria. "La miscellanea laurenziana XXXIII, 31.” Studi sul Boccaccio, VII (1973): 59-124. Print. 
d'Arco Silvio Avalle, "I canzonieri: definizione di genere e problemi di edizione.” La critica del testo. Problemi di metodo ed esperienze di lavoro, Atti del Convegno di Lecce 1984. Roma: Salerno editore, 1985. 363-382. Print.

Di Benedetto, Filippo. "Considerazioni sullo Zibaldone laurenziano del Boccaccio e restauro testuale della prima redazione del 'Faunus.”' Italia Medioevale e Umanistica, XIV (1971): 91-129. Print.

---. "Lo Zibaldone Laurenziano, libro segreto del Boccaccio." VI centenario della morte di Giovanni Boccaccio. Mostra di manoscritti, documenti e edizioni. Firenze, Biblioteca Medicea Laurenziana 22 maggio-31 agosto 1975, a cura del Comitato promotore, vol. I, Manoscritti e documenti, Certaldo, 1975. 117-122. Print.

---. "Presenza di testi minori negli Zibaldoni."Gli Zibaldoni di Boccaccio. $\quad$ Eds. Michelangelo Picone, e Claude Cazalé Bérard. 13-28. Print.

Fiorentino, Francesco. "Infinite reti: la letteratura nell’ipertesto della cultura." Al di là del testo. Critica letteraria e studio della cultura. Ed. Francesco Fiorentino. Macerata: Quodlibet, 2011. 9-62. Print.

Feliziani, Ombretta. "Per l'edizione critica informatizzata dello Zibaldone Laurenziano." Digital Philology and Medieval Texts. Eds. Arianna Ciula, e Francesco Stella. Pisa: Pacini, 2007. 33-63. Print.

Giaveri, Maria Teresa, Grésillon, Almuth, eds. I sentieri della creazione. Tracce traiettorie modelli / Les sentiers de la création. Traces trajectoires modèles, Reggio Emilia: Diabasis, 1994. Print.

Grésillon, Almuth, "Philologie et critique génétique: ressemblances et différences." I Nuovi orizzonti della filologia: ecdotica, critica testuale, editoria scientifica e mezzi informatici elettronici in collaborazione con l'Associazione Internazionale per gli Studi di Lingua e Letteratura italiana (Roma, 27-29 maggio 1998). Roma: Accademia Nazionale dei Lincei, 1999. 53-58. Print.

Hauvette, Henri. "Notes sur des manuscrits autographes de Boccaccio à la Bibliotheque Laurentienne." Mélanges d'Archéologie et d'Histoire, de l'école française de Rome, XIV (1894), 87-145; Etudes sur Boccaccio (1894-1916) con Prefazione di Carlo Pellegrini. Torino: Bottega d'Erasmo, 1968. Print.

Lebrave, Jean-Louis. "L’édition critique au XXIe siècle.” I nuovi orizzonti della filologia. Ecdotica, critica testuale, editoria scientifica e mezzi informatici elettronici. Atti del Convegno Internazionale in collaborazione con l'Associazione Internazionale per gli Studi di Lingua e Letteratura Italiana (Roma, 27-29 maggio 1998). Roma: Accademia Nazionale dei Lincei, 1999. 127-132. Print.

Migne, Jacques Paul. Patrologia Latina. Vol. XXIII. Print.

Mordenti, Raul. Parádosis. A proposito del testo informatico. Roma: Accademia Nazionale dei Lincei-Bardi, 2012. Print. 
---. "La conoscenza in rete come bene comune." Relazione al XV Congresso di Informatica Umanistica della Fondazione "Ezio Franceschini" Onlus, Montevarchi, 22-23 novembre 2007. Testo e senso, 12 (2011), July 30, 2012. Web. <www.testoesenso.it>.

---. Informatica e critica dei testi. Roma: Bulzoni, 2001. Print.

Novati, Francesco. Recensione di Hauvette, Henri. "Notes sur des manuscrits autographes de Boccaccio à la Bibliotheque Laurentienne." Giornale Storico della Letteratura Italiana, XIII (1895), vol. xxv: 422-424. Print.

Orlandi, Tito. Informatica testuale. Teoria e prassi. Roma-Bari: Laterza, 2011. Print.

Petrucci, Armando. "Minuta, autografo, libro d'autore.” Atti del Convegno internazionale: II Libro e il Testo. Eds. Cesare Questa, e Renato Raffaelli. Urbino: Università degli Studi di Urbino, 1984. 397-414. Print.

Picone, Michelangelo, e Cazalé Bérard, Claude, eds. Gli Zibaldoni di Boccaccio. Memoria, scrittura, riscrittura, Atti del Seminario internazionale di Firenze-Certaldo (26-28 aprile 1996). Firenze : Cesati, 1998. Print.

Quaglio, Antonio Enzo. Scienza e mito nel Boccaccio. Padova: Liviana, 1967. Print.

Sabbadini, Remigio. Le scoperte dei codici latini e greci ne’ secoli XIV e XV. Vol. I. Firenze: Sansoni, Firenze 1905. Print.

---. Le scoperte dei codici latini e greci ne’ secoli XIV e XV. Vol. I. Ristampa anastatica con nuove aggiunte e correzioni dell'Autore. Ed. Eugenio Garin. Firenze: Sansoni, 1967. Print.

Zamponi, Stefano. "Genesi e metamorfosi del libro segreto di Boccaccio. Un'indagine fra filologia e codicologia (con un progetto di restauro virtuale)." I nuovi orizzonti della filologia. Ecdotica, critica testuale, editoria scientifica e mezzi informatici elettronici. Convegno internazionale, Roma, 27-29 maggio 1998. Roma: Accademia Nazionale dei Lincei, 1999. 37-51. Print.

Zamponi, Stefano, Pantarotto, Martina, e Tomiello, Antonella. "Stratigrafia dello Zibaldone e della Miscellanea laurenziana." Gli Zibaldoni di Boccaccio. Memoria, scrittura, riscrittura. Eds. Alessandro Picone, e Claude Cazalé Bérard. 181-243. Print.

Zumthor, Paul. La lettre et la voix. Paris: Seuil, 1987. Print. 